\title{
Digital Repository Legacies: A Case Study in Assessing Organizational Trustworthiness
}

Dana Reijerkerk \& Kristen J. Nyitray Stony Brook University Libraries

December 2, 2021 


\section{Abstract}

"Academic libraries rarely discuss cases of digital repositories that do not meet the standards expected of trusted digital repositories.

Implications from inconsistent adherence to technical and professional criteria often surface during migration projects."

In 2020, Stony Brook University Libraries began migrating assets to a mono-repository environment. Persistent historical factors presented challenges to repository trustworthiness.

This case study discusses a survey project to evaluate legacy repository statuses in the contexts of infrastructure, documentation, and staff capacity. It considers a paradigm of organizational accountability in digital asset stewardship and offers insights for reconciling inherited legacies with aspirations to be a trusted repository." 


\section{Introduction}

- This present study was spurred by preliminary planning work at Stony Brook University Libraries (SBUL), an academic research library, in anticipation of migrating assets from a multitude of repositories to a mono-repository environment, DSpace 6.3.

- Digital repository standard: Audit and Certifications of Trustworthy Digital Repositories

- Trustworthy Repositories Audit \& Certification: Criteria and Checklist (TRAC)

- We conducted a survey project designed to appraise digital repository legacies at SBUL with an emphasis on organizational infrastructure using the TRAC Checklist and two digital preservation assessment reports. 


\section{About the University Libraries}

1957: Stony Brook University founded at Oyster Bay, NY (temporary campus)

1963: library opens on the Stony Brook campus

1971: library expansion project completed

late 1990s: developed multiple digital repositories (e.g., DSpace, CONTENTdm)

2015: bepress (Academic Commons)

2016: Omeka

2020: Began migration of digital content to DSpace 6.3

\section{Present}

- No program or system for digital asset management (DAM).

- DRs are maintained separately from the libraries' online integrated discovery catalog (Ex Libris/Alma).

- DRs are used as short-term storage and access systems.

- The IR software bepress is used exclusively to publish faculty scholarly output. 


\section{Research Questions}

1. To what extent do SBUL meet the eighteen benchmarks outlined in

"Appendix 3: Minimum Required Documents" of Trustworthy Repositories Audit

\& Certification: Criteria Checklist (TRAC)?

2. What evidence supports these findings: documentation, transparency, adequacy, and/or measurability?

3. What mitigating actions could advance SBUL toward achieving unmet benchmarks?

4. What opportunities and barriers exist for meeting unmet benchmarks? 


\section{Literature Review}

- Impetus for developing digital repositories

- Standards and best practices

- Management and infrastructure

- Professional development and training considerations 


\section{The Problem:}

\section{Reconciling legacy assets for migration with organizational readiness}

- Library administration announced DSpace version 6.3 would be the platform for all digital assets

- New repository environment:

- DSpace (asset management)

- Omeka (exhibitions)

- Be Press (scholarly works)

- Formed a working group based on organizational reporting structure, not skill sets

- Leading up to this, repositories weren't receiving major system updates and patches

- Pre-existing gaps in training and knowledge 


\section{Methods}

Assessed SBUL for evidence of the 18 minimal required documents specified in TRAC

1. Criteria was taken from Appendix C, "Minimum Required Documents" of the Trustworthy Repositories Audit \& Certification: (TRAC) Criteria Checklist

2. Digital Preservation Coalition's maturity modeling tool, the "Rapid Assessment Model" (DPC RAM) Rating Scale 0-4
a. $0=$ "minimal awareness"
b. 1 = "awareness"
c. 2 = "basic"
d. 3 = "managed"
e. 4 = "optimized"

3. Assigned ratings informed by the findings of two SBUL rapid assessment digital preservation benchmark reports that also used the DPC RAM to measure the state of SBUL's DRs 


\section{Results}

Table 1. SBUL rapid benchmark assessment using the TRAC minimum required documents and the DPC RAM model.

\begin{tabular}{|c|c|c|c|c|c|c|c|}
\hline $\begin{array}{l}\text { TRAC } \\
\text { organization section }\end{array}$ & TRAC sub-section & TRAC citeria & $\begin{array}{l}\text { Examples of TRAC } \\
\text { stipulated documents }\end{array}$ & $\begin{array}{l}\text { DPC RAM } \\
\text { organizational } \\
\text { capability } \\
\text { category }\end{array}$ & $\begin{array}{l}\text { SBUL evidence: } \\
\text { documentation, } \\
\text { transparency, adequacy, } \\
\text { and measurability }\end{array}$ & $\begin{array}{c}\text { SBUL rating: } 0=\text { minimal } \\
\text { awareness, } \\
\begin{array}{c}1=\text { awareness, } 2=\text { basic, } \\
3=\text { managed, } \\
4=\text { optimized }\end{array}\end{array}$ & Suggested mitigating actions \\
\hline $\begin{array}{l}\text { A. Organizational } \\
\text { Infrastructure }\end{array}$ & $\begin{array}{l}\text { A1. Govemance \& } \\
\text { organizational } \\
\text { viability }\end{array}$ & $\begin{array}{l}\text { A1.2 Repository has an appropriate, co } \\
\text { formal succession plan, } \\
\text { contingency plans, and/or } \\
\text { escrow arrangements in place in } \\
\text { case the repository ceases to } \\
\text { operate or the governing or } \\
\text { funding institution substantially } \\
\text { changes its scope. }\end{array}$ & $\begin{array}{l}\text { Contingency plans; } \\
\text { succession plans, } \\
\text { escrow } \\
\text { arrangements (as } \\
\text { appropriate) }\end{array}$ & $\begin{array}{l}\text { Organizational } \\
\text { viability }\end{array}$ & $\begin{array}{l}\text { informal system exit } \\
\text { strategy, automated } \\
\text { data backup to deep } \\
\text { storage; loose } \\
\text { arrangements to retum } \\
\text { data to } \\
\text { content creators }\end{array}$ & 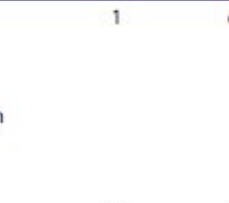 & $\begin{array}{l}\text { develop formal succession plan; } \\
\text { public facing audited annual } \\
\text { financial statements; qeate } \\
\text { financial forecasts with multiple } \\
\text { budget scenarios }\end{array}$ \\
\hline $\begin{array}{l}\text { A. Organizational } \\
\text { Infrastructure }\end{array}$ & $\begin{array}{l}\text { A3. Procedural } \\
\text { accountability \& } \\
\text { policy framework }\end{array}$ & $\begin{array}{l}\text { A3.1 Repository has defined its } \\
\text { designated community(lies) and } \\
\text { associated knowledge base(s) } \\
\text { and has publicly accessible } \\
\text { definitions and policies in place } \\
\text { to dictate how its preservation } \\
\text { service requirements will } \\
\text { be met. }\end{array}$ & $\begin{array}{l}\text { definition of } \\
\text { designated } \\
\text { community(ies), } \\
\text { and policy relating } \\
\text { to service levels }\end{array}$ & $\begin{array}{l}\text { Policy and } \\
\text { strategy }\end{array}$ & $\begin{array}{l}\text { some designated } \\
\text { communities are } \\
\text { defined (e.g., } \\
\text { institutional repository } \\
\text { user community); } \\
\text { some collections have } \\
\text { service-level } \\
\text { agreeements }\end{array}$ & 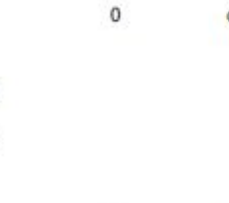 & $\begin{array}{l}\text { create written definition of } \\
\text { designated community(ies); } \\
\text { publish policies detailing SBUL's } \\
\text { preservation strategies }\end{array}$ \\
\hline $\begin{array}{l}\text { A. Organizational } \\
\text { Infrastructure }\end{array}$ & $\begin{array}{l}\text { A3. Procedural } \\
\text { accountability \& } \\
\text { policy framework }\end{array}$ & $\begin{array}{l}\text { A3.3 Repository maintains written } \\
\text { policies that specify the nature } \\
\text { of any legal permissions } \\
\text { required to preserve digital } \\
\text { content over time, and } \\
\text { repository can demonstrate that } \\
\text { these permissions have been } \\
\text { acquired when needed. }\end{array}$ & $\begin{array}{l}\text { policies relating to } \\
\text { legal permissions }\end{array}$ & Legal basis & $\begin{array}{l}\text { informal service } \\
\text { agreements for digital } \\
\text { project content }\end{array}$ & 1 & $\begin{array}{l}\text { implement use contracts and } \\
\text { deposit agreements; specify } \\
\text { which rights transfer for } \\
\text { different types of digital } \\
\text { content; create a policy } \\
\text { statement on required rights for } \\
\text { preservation purposes }\end{array}$ \\
\hline $\begin{array}{l}\text { A. Organizational } \\
\text { Infrastructure }\end{array}$ & $\begin{array}{l}\text { A3. Procedural } \\
\text { accountability \& } \\
\text { policy framework }\end{array}$ & $\begin{array}{l}\text { A3.5 Repository has policies and } \\
\text { procedures to ensure that } \\
\text { feedback from producers and } \\
\text { users is sought and addressed } \\
\text { over time. }\end{array}$ & $\begin{array}{l}\text { policies and } \\
\text { procedures relating } \\
\text { to feedback }\end{array}$ & $\begin{array}{l}\text { Policy and } \\
\text { strategy }\end{array}$ & $\begin{array}{l}\text { no formal process to } \\
\text { collect feedback }\end{array}$ & 0 & $\begin{array}{l}\text { create a workflow to systematically } \\
\text { solicit feedback regarding } \\
\text { software and service adequacy }\end{array}$ \\
\hline $\begin{array}{l}\text { A. Organizational } \\
\text { Infrastructure }\end{array}$ & $\begin{array}{l}\text { A4. Financial } \\
\text { sustainability }\end{array}$ & $\begin{array}{l}\text { A4.3 Repository's financial practices } \\
\text { and procedures are transparent, } \\
\text { compliant with relevant } \\
\text { accounting standards and } \\
\text { practices, and audited by third } \\
\text { parties in accordance with } \\
\text { territorial legal requirements. }\end{array}$ & Financial procedures & $\begin{array}{l}\text { Organizational } \\
\text { viability }\end{array}$ & $\begin{array}{l}\text { Licensing and software } \\
\text { expenditures are } \\
\text { tracked and procured } \\
\text { through University } \\
\text { Procurement and } \\
\text { Library Administration }\end{array}$ & 2 & $\begin{array}{l}\text { create reports of financial and } \\
\text { technical audits, and } \\
\text { certifications; annually audit } \\
\text { financial statements; create } \\
\text { contingency plans }\end{array}$ \\
\hline $\begin{array}{l}\text { A. Organizational } \\
\text { Infrastructure }\end{array}$ & $\begin{array}{l}\text { A5. Contracts, licenses, } \\
\text { \& liabilities }\end{array}$ & $\begin{array}{l}\text { A5.5 If repository ingests digital } \\
\text { content with undear ownership/ } \\
\text { rights, policies are in place to }\end{array}$ & $\begin{array}{l}\text { Policies/procedures } \\
\text { relating to } \\
\text { challenges to rights }\end{array}$ & Legal basis & $\begin{array}{l}\text { practice since } 2016 \text { for } \\
\text { new content to } \\
\text { include }\end{array}$ & 1 & $\begin{array}{l}\text { define required rights, licenses, and } \\
\text { permissions to be obtained } \\
\text { upon deposit; publish legal }\end{array}$ \\
\hline
\end{tabular}




\begin{tabular}{|c|c|c|c|c|c|c|c|}
\hline $\begin{array}{l}\text { TRAC } \\
\text { organization section }\end{array}$ & TRAC sub-section & TRAC aiteria & $\begin{array}{l}\text { Examples of TRAC } \\
\text { stipulated documents }\end{array}$ & $\begin{array}{l}\text { DPC RAM } \\
\text { organizational } \\
\text { capability } \\
\text { category }\end{array}$ & $\begin{array}{l}\text { SBUL evidence: } \\
\text { documentation, } \\
\text { transparency, adequacy, } \\
\text { and measurability }\end{array}$ & $\begin{array}{l}\text { SBUL rating: } 0=\text { minimal } \\
\text { awareness, } \\
\begin{array}{c}1 \text { = awareness, } 2=\text { basic, } \\
3=\text { managed, } \\
4=\text { optimized }\end{array}\end{array}$ & Suggested mitigating actions \\
\hline & & $\begin{array}{l}\text { address liability and challenges } \\
\text { to those rights. }\end{array}$ & $\begin{array}{l}\text { (only if likely to } \\
\text { be needed) }\end{array}$ & & $\begin{array}{l}\text { rightsstatements.org } \\
\text { statements; challenges } \\
\text { managed informally }\end{array}$ & & $\begin{array}{l}\text { liability dause; create and } \\
\text { publish a policy on responding } \\
\text { to challenges }\end{array}$ \\
\hline $\begin{array}{l}\text { B. Digital } \\
\text { Object } \\
\text { Management }\end{array}$ & $\begin{array}{l}\text { B1. Ingest: acquisition } \\
\text { of content }\end{array}$ & B1. Procedures related to ingest. & $\begin{array}{l}\text { Procedures related } \\
\text { to ingest }\end{array}$ & $\begin{array}{l}\text { Policy and } \\
\text { strategy }\end{array}$ & $\begin{array}{l}\text { established process for } \\
\text { ingesting content in } \\
\text { batch; repository-wide } \\
\text { metadata profile that } \\
\text { identifies SIP } \\
\text { properties; repository } \\
\text { ceates checksums } \\
\text { upon ingest (B1.3) }\end{array}$ & c & $\begin{array}{l}\text { create preservation procedures } \\
\text { based on mission statement; } \\
\text { develop and require } \\
\text { submission agreements }\end{array}$ \\
\hline $\begin{array}{l}\text { B. Digital } \\
\text { Object } \\
\text { Management }\end{array}$ & $\begin{array}{l}\text { B2. Ingest: creation of } \\
\text { the } \\
\text { archivable package }\end{array}$ & $\begin{array}{l}\text { B2.10 Repository has a documented } \\
\text { process for testing } \\
\text { understandability of the } \\
\text { information content and } \\
\text { bringing the information } \\
\text { content up to the agreed level } \\
\text { of understandability. }\end{array}$ & $\begin{array}{l}\text { dProcess for testing } \\
\text { understandability }\end{array}$ & $\begin{array}{l}\text { Policy and } \\
\text { strategy }\end{array}$ & $\begin{array}{l}\text { generate persistent, } \\
\text { unique identifiers for } \\
\text { all AlPs; built-in Dublin } \\
\text { Core metadata registry; } \\
\text { built-in file } \\
\text { format registry }\end{array}$ & ; & $\begin{array}{l}\text { investment in staff training to } \\
\text { develop digital preservation } \\
\text { expertise; conduct user- } \\
\text { exoerience testing with the } \\
\text { designated community }\end{array}$ \\
\hline $\begin{array}{l}\text { B. Digital } \\
\text { Object } \\
\text { Management }\end{array}$ & $\begin{array}{l}\text { B4. Archival storage \& } \\
\text { preservation/ } \\
\text { maintenance } \\
\text { of AlPs }\end{array}$ & $\begin{array}{l}\text { B4.1 Repository employs } \\
\text { documented } \\
\text { preservation strategies. }\end{array}$ & Preservation strategies & $\begin{array}{l}\text { s Policy and } \\
\text { strategy }\end{array}$ & $\begin{array}{l}\text { annual preservation } \\
\text { assessment and report; } \\
\text { bitstream preservation } \\
\text { via built- in checksum } \\
\text { ceator and } \\
\text { authenticating tool }\end{array}$ & ; & $\begin{array}{l}\text { ceate a preservation } \\
\text { implementation plan; document } \\
\text { identified preservation risks and } \\
\text { strategies for dealing with } \\
\text { each risk }\end{array}$ \\
\hline $\begin{array}{l}\text { B. Digital } \\
\text { Object } \\
\text { Management }\end{array}$ & $\begin{array}{l}\text { B4. Archival storage \& } \\
\text { preservation/ } \\
\text { maintenance } \\
\text { of AlPs }\end{array}$ & $\begin{array}{l}\text { B4.2 Repository implements/ } \\
\text { responds to strategies for } \\
\text { archival object (i.e., AIP) storage } \\
\text { and migration. }\end{array}$ & $\begin{array}{l}\text { Storage/ } \\
\text { migration } \\
\text { strategies }\end{array}$ & $\begin{array}{l}\text { Policy and } \\
\text { strategy }\end{array}$ & $\begin{array}{l}\text { create checksums for each } \\
\text { ingested digital object }\end{array}$ & c & $\begin{array}{l}\text { create a regular schedule to check } \\
\text { fixity information; document } \\
\text { how AlPs and fixity information } \\
\text { are kept separate; create logs of } \\
\text { fixity checks }\end{array}$ \\
\hline $\begin{array}{l}\text { B. Digital } \\
\text { Object } \\
\text { Management }\end{array}$ & $\begin{array}{l}\text { B6. } \\
\text { Access } \\
\text { management }\end{array}$ & $\begin{array}{l}\text { B6.2 Repository has implemented a } \\
\text { policy for recording all access } \\
\text { actions (includes requests, } \\
\text { orders etc.) that meet the } \\
\text { requirements of the repository } \\
\text { and information } \\
\text { producers/depositors. }\end{array}$ & $\begin{array}{l}\text { Policy for recording } \\
\text { access actions }\end{array}$ & $\begin{array}{l}\text { Policy and } \\
\text { strategy }\end{array}$ & $\begin{array}{l}\text { no established access } \\
\text { policies for library- } \\
\text { created assets; use of } \\
\text { password } \\
\text { authentication for } \\
\text { content creators }\end{array}$ & re & $\begin{array}{l}\text { record access actions; create logs } \\
\text { and audit trails of } \\
\text { access requests }\end{array}$ \\
\hline $\begin{array}{l}\text { B. Digital } \\
\text { Object } \\
\text { Management }\end{array}$ & $\begin{array}{l}\text { B6. } \\
\text { Access } \\
\text { management }\end{array}$ & $\begin{array}{l}\text { B6.4 Repository has documented } \\
\text { and implemented access } \\
\text { policies (authorization rules, }\end{array}$ & Policy for access & $\begin{array}{l}\text { Policy and } \\
\text { strategy }\end{array}$ & $\begin{array}{l}\text { use of embargo item and } \\
\text { collection functions }\end{array}$ & 2 & $\begin{array}{l}\text { areate written policies on third } \\
\text { party deposit arrangements; }\end{array}$ \\
\hline
\end{tabular}




\begin{tabular}{|c|c|c|c|c|c|c|c|}
\hline $\begin{array}{l}\text { TRAC } \\
\text { organization section }\end{array}$ & TRAC sub-section & TRAC aiteria & $\begin{array}{c}\text { Examples of TRAC } \\
\text { stipulated documents }\end{array}$ & $\begin{array}{l}\text { DPC RAM } \\
\text { organizational } \\
\text { capability } \\
\text { category }\end{array}$ & $\begin{array}{l}\text { SBUL evidence: } \\
\text { documentation, } \\
\text { transparency, adequacy, } \\
\text { and measurability }\end{array}$ & $\begin{array}{c}\text { SBUL rating: } 0=\text { minimal } \\
\text { awareness, } \\
\begin{array}{c}1=\text { awareness, } 2=\text { basic, } \\
3=\text { managed } \\
4=\text { optimized }\end{array}\end{array}$ & Suggested mitigating actions \\
\hline & & $\begin{array}{l}\text { authentication requirements) } \\
\text { consistent with deposit } \\
\text { agreements for stored objects. }\end{array}$ & & & & & $\begin{array}{l}\text { ceate definitions of service } \\
\text { levels and permitted uses }\end{array}$ \\
\hline $\begin{array}{l}\text { C. Technologies, } \\
\text { Technical } \\
\text { Iffrastructure, } \\
\text { \& Security }\end{array}$ & $\begin{array}{l}\text { C1.7 Repository has } \\
\text { defined processes } \\
\text { for storage media } \\
\text { and/or hardware } \\
\text { change (e.g. } \\
\text { refreshing, } \\
\text { migration). }\end{array}$ & $\begin{array}{l}\text { C1.7 Repository has defined } \\
\text { processes for storage media } \\
\text { and/or hardware change (e.g. } \\
\text { refreshing, migration). }\end{array}$ & $\begin{array}{l}\text { Processes for } \\
\text { media change }\end{array}$ & $\pi$ capability & $\begin{array}{l}\text { limited processes for } \\
\text { assessment and } \\
\text { migratitons; } \\
\text { no refreshing }\end{array}$ & c & $\begin{array}{l}\text { create documentation for migration } \\
\text { processes; embed systemic and } \\
\text { scheduled work for assessing } \\
\text { refreshing, migrations, and } \\
\text { storage capacity; create policies } \\
\text { related to hardware support, } \\
\text { maintenance, and replacement; } \\
\text { document hardware } \\
\text { manufacturer's expected } \\
\text { support life cycles }\end{array}$ \\
\hline $\begin{array}{l}\text { c. Technologies, } \\
\text { Technical } \\
\text { Iffratructure, } \\
\text { \& Security }\end{array}$ & $\begin{array}{l}\text { C1.8 Repository has a } \\
\text { documented } \\
\text { change } \\
\text { management } \\
\text { process that } \\
\text { identifies changes } \\
\text { to citical processes } \\
\text { that potentially } \\
\text { affect the } \\
\text { repository's ability } \\
\text { to comply with its } \\
\text { mandatory } \\
\text { responsibilities. }\end{array}$ & $\begin{array}{l}\text { C1.8 Repository has a documented } \\
\text { change management process } \\
\text { that identifies changes to catical } \\
\text { processes that potentially affect } \\
\text { the repository's ability to } \\
\text { comply with its mandatory } \\
\text { responsibilities. }\end{array}$ & $\begin{array}{l}\text { Change } \\
\text { management } \\
\text { process }\end{array}$ & $\begin{array}{l}\text { Continous } \\
\text { improvement }\end{array}$ & $\begin{array}{l}\text { mechanism shows the } \\
\text { automated process of } \\
\text { system changes using } \\
\text { the Graphical } \\
\text { User Interface }\end{array}$ & ( & $\begin{array}{l}\text { document the change management } \\
\text { process; conduct a risk } \\
\text { assessment of the change } \\
\text { management process }\end{array}$ \\
\hline $\begin{array}{l}\text { C. Technologies, } \\
\text { Technical } \\
\text { Iffrastructure, } \\
\text { \& Security }\end{array}$ & $\begin{array}{l}\text { C1.9 Repository has a } \\
\text { process for testing } \\
\text { the effect of critical } \\
\text { changes to } \\
\text { the system. }\end{array}$ & $\begin{array}{l}\text { C1.9 Repository has a process for } \\
\text { testing the effect of citical } \\
\text { changes to the system. }\end{array}$ & $\begin{array}{l}\text { Gitical change } \\
\text { test process }\end{array}$ & $\pi$ capability & $\begin{array}{l}\text { some system upgrades } \\
\text { are done in a test } \\
\text { environment but are } \\
\text { undocumented }\end{array}$ & 2 & $\begin{array}{l}\text { document results in a report; } \\
\text { analyze the impact of a } \\
\text { process change }\end{array}$ \\
\hline \multirow[t]{2}{*}{$\begin{array}{l}\text { C. Technologies, } \\
\text { Technical } \\
\text { Inff astructure, } \\
\text { \& Security }\end{array}$} & $\begin{array}{l}\text { C1.10 Repository has a } \\
\text { process to react to } \\
\text { the availability of } \\
\text { new software } \\
\text { security updates } \\
\text { based on a risk- } \\
\text { benefit assessment }\end{array}$ & $\begin{array}{l}\text { C1.10 Repository has a process to } \\
\text { react to the avallability of new } \\
\text { software security updates based } \\
\text { on a risk-benefit assessment. }\end{array}$ & $\begin{array}{l}\text { Security } \\
\text { update process }\end{array}$ & I capability & $\begin{array}{l}\text { there is evidence of } \\
\text { update processes in } \\
\text { the Graphical User } \\
\text { Interface in the Really } \\
\text { Simple Syndication } \\
\text { (RSS) feed }\end{array}$ & 2 & $\begin{array}{l}\text { document system update } \\
\text { installations; create a } \\
\text { risk register }\end{array}$ \\
\hline & & & & 匹 capability & inventory of hardware & 2 & \\
\hline
\end{tabular}


Table 1. Continued.

\begin{tabular}{|c|c|c|c|c|c|c|c|}
\hline $\begin{array}{l}\text { TRAC } \\
\text { organization section }\end{array}$ & TRAC sub-section & TRAC criteria & $\begin{array}{l}\text { Examples of TRAC } \\
\text { stipulated documents }\end{array}$ & $\begin{array}{l}\text { DPC RAM } \\
\text { organizational } \\
\text { capability } \\
\text { category }\end{array}$ & $\begin{array}{l}\text { SBUL evidence: } \\
\text { documentation, } \\
\text { transparency, adequacy, } \\
\text { and measurability }\end{array}$ & $\begin{array}{c}\text { SBUL rating: } 0=\text { minimal } \\
\text { awareness, } \\
\begin{array}{c}1=\text { awareness, } 2=\text { basic, } \\
3=\text { managed, } \\
4=\text { optimized }\end{array}\end{array}$ & Suggested mitigating actions \\
\hline $\begin{array}{l}\text { C. Technologies, } \\
\text { Technical } \\
\text { Infrastructure, } \\
\text { \& Security }\end{array}$ & $\begin{array}{r}\text { C2. Appropriate } \\
\text { technologies }\end{array}$ & $\begin{array}{l}\text { C2.1 Repository has hardware } \\
\text { technologies appropriate to the } \\
\text { services it provides to its } \\
\text { designated community(ies) and } \\
\text { has procedures in place to } \\
\text { receive and monitor } \\
\text { notifications, and evaluate when } \\
\text { hardware technology changes } \\
\text { are needed. }\end{array}$ & $\begin{array}{l}\text { Process to monitor } \\
\text { required changes } \\
\text { to hardware }\end{array}$ & & & & $\begin{array}{l}\text { maintain currency of hardware and } \\
\text { of new technology changes }\end{array}$ \\
\hline $\begin{array}{l}\text { C. Technologies, } \\
\text { Technical } \\
\text { Infrastructure, } \\
\text { \& Security }\end{array}$ & $\begin{array}{l}\text { C2. Appropriate } \\
\text { technologies }\end{array}$ & $\begin{array}{l}\text { C2.2 Repository has software } \\
\text { technologies appropriate to the } \\
\text { services it provides to its } \\
\text { designated community(ies) and } \\
\text { has procedures in place to } \\
\text { receive and monitor } \\
\text { notifications, and evaluate when } \\
\text { software technology changes } \\
\text { are needed. }\end{array}$ & $\begin{array}{l}\text { Process to monitor } \\
\text { required changes } \\
\text { to software }\end{array}$ & IT capability & inventory of software & it & $\begin{array}{l}\text { invest in the access and } \\
\text { preservation software required } \\
\text { for the designated community; } \\
\text { develop procedures to recive } \\
\text { and monitor notification; } \\
\text { maintain currency of } \\
\text { software technology }\end{array}$ \\
\hline
\end{tabular}




\section{Discussion}

- Organizational infrastructure

- "The retrieval and functionality of digital assets is dependent on sound organizational planning and an infrastructure driven by well-formed written policies and procedures, and with an institutional investment in and commitment to its sustainability and growth."

- Digital object management

- "Digital object management would benefit greatly from participation of current staff members who possess technical expertise, credentials in the domain of digital archives, and historical information about legacy collections."

- Technologies, technical infrastructure, and security

- "Considerations for technologies, technical infrastructure, and security emphasize the critical processes for maintaining compliance of systems, assessing hardware and software, and creating procedures to survey and maintain technology." 


\section{Conclusion}

Integrate organizational accountability in the paradigm of digital asset stewardship.

- Balance aspirations with capacity; demonstrates a commitment to the standards that embody a TDR.

- Libraries can lead and actively contribute to programs that ensure the long-term preservation of cultural heritage and research.

- Sustainability of digital assets depends on investment in resources and requires reaffirming commitments to maintain them.

- Connect digital asset preservation to mission-driven goals and objectives.

- Institutions facing DR environments similar to SBUL can model this study:

- use a modified version of the TRAC list of minimum required documents and DPC RAM rating scale.

- fuller design process could then ensue, particularly at organizations where little documentation and historical information exists. 


\section{Article}

Nyitray, Kristen J. \& Reijerkerk, D. (2021)

"Digital Repository Legacies: A Case Study in Assessing Organizational Trustworthiness,"

Journal of Library Administration 61 (7), https://doi.org/10.1080/01930826.2021.1972729. 